\title{
Correlação entre a Idade Materna, Paridade, Gemelaridade, Sindrome Hipertensiva e Ruptura Prematura de Membranas e a Indicação de Parto Cesáreo
}

The Influence of Maternal Age, Parity, Twin Pregnancy, Hypertensive Syndrome and Premature Rupture of Membranes on the Indication for Cesarean Section

Simone Angélica Leite de Carvalho Silva Cabral, Cícero Ferreira Fernandes Costa, Sérgio Fernandes Cabral Júnior

\section{RESUM0}

\begin{abstract}
Objetivo: verificar a contribuição da idade materna, paridade, gemelaridade, sindrome hipertensiva, ruptura prematura das membranas como fator de risco para cesárea.

Métodos: após aprovação do Comitê de Ética em Pesquisa da Maternidade Professor Monteiro de Morais, situada em Recife-PE, realizou-se estudo de caso-controle com 3919 gestantes, sem antecedente de duas ou mais cesáreas, que deram à luz concepto vivo, com idade gestacional igual ou superior a 28 semanas, peso mínimo de $1.000 \mathrm{~g}$, em apresentação cefálica, no período de 1 de setembro de 1999 a 31 de agosto de 2000. No grupo caso foram incluídas mulheres submetidas a operação cesariana e no grupo controle, a parto vaginal. Com os dados constantes dos prontuários neonatais e obstétricos, realizou-se análise multivariada por regressão logística, buscando a equação matemática que relacione a probabilidade de ocorrência de cesárea decorrente de mais de uma variável independente atuando como fator de risco, utilizando odds ratio e intervalo de confiança de 95\% (IC 95\%), consideradas as variáveis: idade materna, paridade, gemelaridade, síndrome hipertensiva e ruptura prematura das membranas.

Resultados: as chances de cesárea foram aumentadas em 8,3 vezes (OR = 8,3; IC 95\%: 3,719,1) na gemelaridade, 3,4 na síndrome hipertensiva (OR = 3,4; IC 95\%: 2,9-4,0), 1,9 na primiparidade $(O R=1,9$; IC 95\% : 1,8-2,0), 1,5 na idade superior a 34 anos $(O R=1,5 ;$ IC 95\%: 1,2-1,8) e 1,2 na presença de ruptura prematura das membranas (OR = 1,2; IC 95\%: 1,0-1,4). Conclusões: ruptura prematura das membranas, idade superior a 34 anos, primiparidade, síndrome hipertensiva e gemelaridade constituíram fatores de risco para cesárea.
\end{abstract}

PALAVRAS-CHAVE: Cesariana. Hipertensão. Ruptura prematura de membranas. Gemelaridade.

\section{Introdução}

A operação cesariana é hoje reconhecidamente a tocurgia, cujas taxas ascendentes no mundo e no Brasil fizeram com que fosse conside-

Maternidade Prof. Monteiro de Morais - Recife - PE Correspondência:

Simone Angélica Leite de Carvalho Silva Cabral

Rua Capitão Sampaio Xavier n ${ }^{\circ} 420$ apto 1801 - Rosarinho 52050-210 - Recife - PE

Fone: (81) 3242-5276

e-mail: cabralrec@terra.com.br rada epidêmica pelas autoridades de saúde ${ }^{1}$. De acordo com o Ministério da Saúde ${ }^{2,3}$, a taxa de cesárea no Brasil foi de 39,2\% em 1998, tendo sido a maior a observada no Estado do Mato Grosso $(59,2 \%)$. Essa tendência também ocorreu em Recife-PE, onde houve aumento da incidência de parto cesáreo de $17,2 \%$ para $28,5 \% 4$.

Apesar de ser procedimento amplamente utilizado, a operação cesariana não é isenta de complicações. Atualmente, é bem definida sua associação com maior freqüência de infecção e de hemorragia maternas, além de maiores índi- 
ces de prematuridade e de síndrome do desconforto respiratório do recém-nascido, quando comparada ao parto transpelviano ${ }^{5.6}$.

Por outro lado, levando-se em consideração o aperfeiçoamento dessa cirurgia através dos tempos e sua conseqüente melhora no quesito segurança, passou a ser utilizada com a intenção de diminuir a morbidade e mortalidade perinatais ${ }^{7}$. Diante disso, suas indicações, antes praticamente restritas a distócia mecânica ${ }^{7}$, foram ampliadas e, hoje, incluem: sofrimento fetal, apresentação pélvica, hemorragia anteparto, doença hipertensiva específica da gravidez, gemelaridade, diabetes e cesárea de repetição, esta última tida, na atualidade, como a principal indicação para essa via de parto no Brasile ${ }^{8-12}$. Já a ruptura prematura das membranas, na literatura, parece não constituir fator de risco para cesárea ${ }^{13-15}$. Com o objetivo de avaliar a determinação da tomotócia, algumas características epidemiológicas vêm sendo estudadas, tais como idade materna ${ }^{16-18}$ e paridade ${ }^{19,20}$.

O conhecimento e a identificação adequada dos fatores de risco para operação cesariana são de fundamental importância, uma vez que nos permitiriam planejar as ações de saúde voltadas à prevenção de morbidades maternas intra e pósparto. O presente estudo foi realizado com o objetivo de determinar a influência de idade materna, paridade, gemelaridade, presença de ruptura prematura das membranas e de sindrome hipertensiva como fatores de risco para cesárea.

\section{Pacientes e Métodos}

Em estudo retrospectivo, do tipo caso-controle, foram analisadas, por meio dos prontuários obstétricos e neonatais, as informações referentes à gravidez e ao parto de 3919 gestantes, que deram à luz na Maternidade Prof. Monteiro de Morais (Recife - PE), no período de 1 de setembro de 1999 a 31 de agosto de 2000, concepto vivo com peso maior que 1000 g, em apresentação cefálica. Foram excluídas as cesáreas realizadas por indicação de iteratividade. A indicação do parto cesáreo obedeceu ao preconizado na literatura ${ }^{21,22}$ e no protocolo do serviço.

As variáveis estudadas foram: via de parto, categorizada como cesáreo ou vaginal; idade materna, caracterizada como o tempo de vida, em anos, registrado no prontuário da gestante, classificada como adolescente, quando a idade fosse inferior a 20 anos, adulta jovem, com idade entre
20 e 34 anos, e adulta, com idade superior a 34 anos; a paridade, conceituada como o número de parturições da gestante, anteriores ao parto tema da presente pesquisa. Foi categorizada como primípara aquela que nunca houvera parido e multipara, a gestante com história de qualquer número de partos anteriores; a gemelaridade foi conceituada como o registro da existência de dois conceptos na gestação em estudo, categorizada em presente e ausente, quando se tratava de gestação única; ruptura prematura de membranas, caracterizada pelo registro em prontuário de história clínica e confirmação ao exame especular de perda de líquido amniótico anterior ao início do trabalho de parto, tendo sido categorizada como presente ou ausente; o diagnóstico de síndrome hipertensiva foi obtido pelo registro no prontuário médico de tensão arterial sistólica igual ou superior a $140 \mathrm{mmHg}$ e diastólica igual ou superior a $90 \mathrm{mmHg}$, após duas aferições com intervalo de quatro a seis horas, categorizada como presente ou ausente.

Os dados foram processados com o auxílio do programa Epi-Info, versão 6.04d, de 14 de junho de 2001, do Centers for Disease Control \& Prevention, da Organização Mundial de Saúde ${ }^{23}$, e analisados por meio do teste $\chi^{2}$, em nível de significância de 0,05, na análise bivariada, procedendo-se à crítica das variáveis segundo critérios de associação biológica. Na análise multivariada adotou-se regressão logística para determinar a equação matemática que relaciona probabilidade de ocorrência de cesárea decorrente de mais de uma variável independente atuando como fator de risco, pelo cálculo do odds ratio (OR), com intervalo de confiança de 95\% (IC 95\%). O trabalho foi realizado após aprovação do Comitê de Ética em Pesquisa da instituição.

\section{Resultados}

O parto de 2833 gestantes ocorreu por via vaginal $(72,3 \%)$, ao passo que para $1086(27,7 \%)$, houve indicação de cesárea. As idades variaram entre 12 e 48 anos (média igual a 23,0 \pm 5,9 anos). Foram classificadas como primíparas $1845(47,1 \%)$ gestantes, ao passo que 2066 (52,9\%) eram multíparas. Nasceram 3956 conceptos, sendo 3882 (98,1\%) em gestação única e 74 (1,9\%), em gemelar. Na internação, houve diagnóstico de ruptura prematura das membranas em 472 (12,0\%) gestantes e sindrome hipertensiva em 794 (20,3\%)(Tabela 1). 
Tabela 1 - Distribuição por idade e outras variáveis obstétricas das 3919 gestantes incluídas.

\begin{tabular}{|c|c|c|}
\hline Variáveis & Número de pacientes & $\%$ \\
\hline \multicolumn{3}{|l|}{ Via de parto } \\
\hline Cesáreo & 1086 & 27,7 \\
\hline Vaginal & 2833 & 72,3 \\
\hline \multicolumn{3}{|l|}{ Idade } \\
\hline$\leq 19$ & 1258 & 32,1 \\
\hline $20-34$ & 2469 & 63,0 \\
\hline$\geq 35$ & 192 & 4,9 \\
\hline \multicolumn{3}{|l|}{ Paridade } \\
\hline Primiparidade & 1845 & 47,1 \\
\hline Multiparidade & 2066 & 52,9 \\
\hline \multicolumn{3}{|l|}{ Gemelaridade } \\
\hline Presente & 37 & 1,0 \\
\hline Ausente & 3882 & 99,0 \\
\hline \multicolumn{3}{|c|}{ Ruptura prematura das membranas } \\
\hline Presente & 472 & 12,0 \\
\hline Ausente & 3447 & 88,0 \\
\hline \multicolumn{3}{|c|}{ Síndromes hipertensivas } \\
\hline Presente & 794 & 20,3 \\
\hline Ausente & 3125 & 79,7 \\
\hline
\end{tabular}

As gestantes com idade igual ou maior que 35 anos foram mais freqüentes no grupo de cesárea (70: $6,5 \%)$ do que no grupo de parto vaginal (122: 4,3\%) e essa diferença foi significativa $\left(\chi^{2}=7,86, p=0,02\right)$ (Tabela 2). Classificando as gestantes em dois grupos quanto à idade - um constituído por mulheres com idades entre $12 \mathrm{e}$ 34 anos, e outro, composto por gestantes entre 35 e 48 anos, detectou-se que a idade igual ou maior que 35 anos associou-se ao aumento de risco de cesárea $(\mathrm{OR}=1,5 ; \mathrm{IC}$ 95,\%: 1,2-1,8) (Tabela 3).

Tabela 2 - Distribuição da via de parto de 3.919 gestantes segundo as faixas etárias.

\begin{tabular}{lrrrr} 
Faixas etárias & \multicolumn{4}{c}{ Via de parto } \\
& \multicolumn{2}{c}{ Cesárea } & \multicolumn{2}{c}{ Vaginal } \\
\hline$\leq 19$ & 338 & 31,1 & 990 & 32,5 \\
$20-34$ & 678 & 62,4 & 1791 & 63,2 \\
$\geq 35$ & 70 & 6,5 & 122 & 4,3 \\
Total & 1086 & 100,0 & 2833 & 100,0 \\
\hline$\chi^{2}=7,86 ;$ g.l. $=2 ; p=0,02$ & & & &
\end{tabular}

Detectou-se associação significativa entre via de parto e paridade. Houve maior freqüência de primíparas $(58,7 \%)$ no grupo de cesárea do que no grupo de parto vaginal $(42,6 \%)$. A primiparidade se associou ao aumento do risco de a gestante ser submetida à cesárea $(\mathrm{OR}=1,9$; IC 95,\%:1,8-
2,0 ). Constatou-se que a gemelaridade constituiu aumento de risco para parto cesáreo $(\mathrm{OR}=8,3$; IC 95\%: 3,7-19,1) (Tabela 3).

Aproximadamente metade das mulheres com síndrome hipertensiva foi submetida à cesárea. A síndrome hipertensiva foi mais freqüente no grupo de cesárea do que no grupo de parto vaginal e essa diferença foi significativa, tendo a presença de sindrome hipertensiva como fator de risco $(\mathrm{OR}=$ 3,4; IC 95\%: 2,9-4,0) (Tabela 3).

Na Tabela 3 observa-se que a ruptura prematura de membranas foi mais freqüente no grupo de cesárea. No entanto, esta diferença não foi significativa. A ocorrência de ruptura prematura das membranas foi fator de risco para parto cesáreo $(\mathrm{OR}=1,2$ IC 95\%: 1, 1-1,4).

\section{Discussão}

A incidência de cesárea no atual estudo foi $27,7 \%$. No entanto, no presente estudo, foram excluídas as cesáreas com indicação de iteratividade. A taxa pode ser considerada semelhante, mas é alta em relação aos $15 \%$ recomendados pela $\mathrm{OMS}^{1}$. Isso se deve ao fato de a Maternidade ser reconhecida como instituição-escola de nível terciário, dotada de enfermaria para gravidez de alto risco, recebendo casos de alta complexidade ou graves, referenciados de maternidades de municípios do interior do Estado.

A associação de idade avançada e cesárea também foi encontrada por outros autores. Isto pode ser explicado pelo fato de estas pacientes estarem mais sujeitas a intercorrências clínicas ${ }^{16-18}$. Yuan et al. ${ }^{18}$ verificaram que gestantes com mais de 35 anos de idade apresentaram risco para cesárea de 2,1 , provavelmente devido a uma monitorização mais próxima dessas pacientes. Os autores consideraram mais seguro submeter as mesmas ao parto cesáreo. Para Scholz et al. ${ }^{16}$, esta tendência também foi observada entre as gestantes com mais de 40 anos de idade, devido, principalmente, à maior incidência de complicações obstétricas.

Amini et al. ${ }^{17}$ constataram que a proporção de cesariana primária entre as adolescentes de 12 a 15 anos foi $11,6 \%$, significativamente mais alta que os 9,4\% para aquelas com idade entre 16 a 19 anos e os 10,2\%, para as adultas. No entanto, os autores notaram que ao longo do período de 10 a 15 anos do estudo, houve declínio na proporção dos partos entre as adolescentes, possivelmente devido a melhor educação sexual, planejamento familiar e infertilidade associada a doenças sexualmente transmitidas. 
Tabela 3 - Distribuição da via de parto de 3919 gestantes segundo grupos etários, paridade, gemelaridade, síndrome hipertensiva e ruptura prematura das membranas.

\begin{tabular}{|c|c|c|c|c|c|c|}
\hline \multirow{3}{*}{ Variáveis } & \multirow{3}{*}{ Categorias } & \multicolumn{4}{|c|}{ Via de parto } & \multirow{3}{*}{$\begin{array}{c}\text { OR } \\
\text { IC } 95 \%\end{array}$} \\
\hline & & \multicolumn{2}{|c|}{ Cesárea } & \multicolumn{2}{|c|}{ Vaginal } & \\
\hline & & $\mathrm{n}$ & $\%$ & $\mathrm{n}$ & $\%$ & \\
\hline \multirow[t]{2}{*}{ Grupos etários } & 20 a 34 anos & 70 & 6,5 & 122 & 4,3 & 1,5 \\
\hline & $\geq 35$ anos & 1016 & 93,5 & 2711 & 95,7 & $1,2-1,8$ \\
\hline \multirow[t]{2}{*}{ Paridade } & Primiparidade & 638 & 58,7 & 1207 & 42,6 & 1,9 \\
\hline & Multiparidade & 448 & 41,3 & 1618 & 57,4 & $1,8-2,0$ \\
\hline \multirow[t]{2}{*}{ Gemelaridade } & Presente & 28 & 2,6 & 9 & 0,3 & 8,3 \\
\hline & Ausente & 1058 & 97,4 & 2824 & 99,7 & $3,7-19,1$ \\
\hline \multirow[t]{2}{*}{ Síndrome hipertensiva } & Presente & 391 & 36,0 & 403 & 14,2 & 3,4 \\
\hline & Ausente & 695 & 64,0 & 2430 & 85,8 & $2,9-4,0$ \\
\hline \multirow[t]{2}{*}{ Ruptura prematura das membranas } & Presente & 148 & 13,6 & 324 & 11,4 & 1,2 \\
\hline & Ausente & 938 & 86,4 & 2509 & 88,6 & $1,1-1,4$ \\
\hline Total & & 1086 & 100,0 & 2833 & 100,0 & \\
\hline
\end{tabular}

Os dados do presente estudo confirmam aqueles da literatura ${ }^{16,18}$ quanto à associação entre idade materna e via de parto, mesmo considerando que a metodologia empregada pelos autores ${ }^{16,18}$ não detalhou os critérios empregados nas indicações de parto cesáreo. Na Maternidade Prof. Monteiro de Morais, por ser instituição-escola, os critérios para indicação de cesárea são rigorosos e alvo de supervisão técnica.

Entre primíparas, Seyb et al. ${ }^{19}$ verificaram taxa de cesárea de $7,8 \%$, com trabalho de parto espontâneo, $17,5 \%$ com parto induzido eletivamente, e 17,7\% com parto cesáreo induzido por indicação médica, identificado como principal fator de risco.

No presente estudo, as primíparas foram mais freqüentes no grupo de cesárea, tendo a condição de primiparidade constituído fator de risco para essa via de parto, o mesmo encontrado em outro estudo ${ }^{19}$. Podem-se aventar duas explicações para essa diferença: a primeira é que não se identificaram quais as pacientes que tiveram o parto induzido; a segunda se refere à interferência da imaturidade do colo uterino sobre o risco de cesárea $^{20}$. De acordo com Abu-Heija e Chalabi ${ }^{20}$, as multíparas, apesar de serem mais idosas e terem mais intercorrências clínicas, não apresentam maior incidência de cesariana, mesmo aquelas que pariram mais de 10 vezes. Essa correlação também foi revelada no presente estudo, em que as multíparas foram mais freqüentes no grupo de parto vaginal.

Com o estabelecimento de critérios rigorosos para indicação de cesárea, constantemente discutidos e atualizados em reunião clínica e obedecidos por decisão pactuada, nos casos de alto risco, assim como com a introdução do partograma, a partir de maio de 2000 , recurso bastante usado em nosso serviço e recomendado pelo Ministério da Saúde, é possível diagnosticar alterações do trabalho de parto e tomar condutas adequadas para a correção desses desvios, contribuindo para evitar a realização de cesáreas desnecessárias ${ }^{1}$.

A associação encontrada no atual estudo entre gemelaridade e cesárea também foi descrita por vários autores ${ }^{8,11}$. Persad et al. ${ }^{11}$, após avaliação de todos os partos gemelares, ocorridos num período de 20 anos, observaram aumento não só na taxa de cesariana eletiva como, também, na taxa de parto vaginal combinado com cesárea para o segundo gemelar. No presente estudo, não foi observada ocorrência dessa última modalidade de parto e a gestação gemelar mostrou-se o mais importante fator de risco para cesárea, possivelmente devido à prática menos freqüente de manobras de versão fetal ${ }^{11}$, verificadas no serviço.

A presença de hipertensão arterial associada à gravidez, nas suas diversas formas clínicas, está associada ao maior risco de operação cesariana $^{8-10}$. Edwards e Witter ${ }^{\natural}$, em estudo retrospectivo, verificaram que o risco de cesárea nas pacientes com pré-eclâmpsia foi o dobro daquelas sem hipertensão arterial associada à gravidez, sendo que $21,1 \%$ das pacientes foram submetidas à cesariana apenas por indicação da pré-eclâmpsia, principalmente quando a forma grave da doença estava presente. Os dados do presente estudo confirmam os da literatura, porém não foi possível 
estabelecer a categorização das formas de apresentação dessa doença e sua correlação com a via de parto. Independentemente do tipo de hipertensão, o maior risco de cesárea, possivelmente, deveu-se à urgência em interromper a gestação, a fim de minimizar complicações a ela associadas.

A maioria dos estudos publicados não considera a ruptura prematura das membranas como fator de risco para a tomotócia ${ }^{13-15}$. Todavia, grande parte dos autores apenas investigou o risco de cesárea, comparando os dados entre as pacientes com essa intercorrência em diferentes formas de conduta, de expectação, medicamentosa e cirúrgica. Na presente análise, a comparação do risco de cesárea foi feita exclusivamente entre pacientes com e sem ruptura prematura das membranas, abordagem não encontrada na literatura.

Os dados do presente trabalho sugerem que alguns fatores podem estar contribuindo para a associação entre ruptura prematura das membranas e parto cesáreo como, por exemplo, a imaturidade do colo uterino.

No presente estudo gemelaridade, síndrome hipertensiva, primiparidade, idade materna superior a 34 anos e presença de ruptura prematura de membranas constituíram fator de risco para operação cesariana, apesar de não se poder descartar a possibilidade de interação de outros fatores que influenciaram a indicação de cesariana. A realização de estudos prospectivos (coorte) é necessária, para manter vigilância constante e de identificar possiveis mudanças no perfil epidemiológico das pacientes submetidas à cesariana.

\section{ABSTRACT}

Purpose: to verify the contribution of maternal age, parity, twin pregnancy, hypertensive syndrome, and premature rupture of membranes as risk factors for cesarean section. Methods: after approval by the Ethics in Research Committee of the "Maternidade Professor Monteiro de Morais"-Recife, PE-Brazil, for a case control study, the authors analyzed data from 3919 pregnant women, without two or more prior cesarean sections, who gave birth to alive newborns with gestational age equal to or more than 28 weeks, weighing at least 1,000 g, on cephalic presentation, from September 1, 1999 to August 31, 2000. The case group included women submitted to cesarean section and the control group included women submitted to vaginal delivery. With the data collected from obstetric and neonatal reports, the authors performed multivariate analysis by logistic regression to determine a mathematical equation that associates cesarean probability due to more than one independent variable acting as risk factor, determining odds ratio with a confidence interval of
95\% (95\% CI), for the variables: maternal age, parity, twin pregnancy, hypertensive syndrome, and premature rupture ofmembranes.

Results: the chances for cesarean section significantly increased 8.3 times in twin pregnancy $(O R=8.3 ; 95 \%$ CI: 3.7-19.1), 3.4 in hypertensive syndrome $(O R=3.4 ; 95 \% \mathrm{CI}$ : 2.9-4.0), 1.9 in primiparity $(O R=1.9 ; 95 \% C I: 1.8-2.0), 1.5$ in maternal age over 34 years $(O R=1.5 ; 95 \%$ CI: 1.2-1.8), and 1.2 in the presence of premature rupture of membranes $(O R=1.2 ; 95 \%$ CI: $1.0-1.4)$.

Conclusions:the riskfor cesarean section was greater in the presence of premature rupture of membranes, maternal age greater than 34 years, primiparity, hypertensive syndrome, and twin pregnancy.

KEYWORDS: Cesarean section. Hypertension. Premature rupture of membranes. Twin pregnancy.

\section{Referências}

1. Ministério da Saúde. Secretaria de Políticas de Saúde. Área Técnica de Saúde da Mulher. Parto, aborto e puerpério: assistência humanizada à mulher. Brasília: Ministério da Saúde; 2001. p.32-7.

2. Ministério da Saúde. Funasa. CENEPI. Sistema de Informações sobre Nascidos Vivos (SINASC). [citado 11 set 2001]. Disponivel em: http:// www.datasus.gov.br/cgi/tabcgi.exe IDB2000/f08.def.

3. Ministério da Saúde. SE. DATASUS. Sistema de Informações Hospitalares do SUS (SIH/SUS). [citado 11 set 2001]. Disponivel em: http:// www.datasus.gov.br/cgi/tabcgi.exe IDB2000/f09.def

4. Costa CFF, Costa HLF, Albuquerque PC. Operação cesariana: incidência e indicações. Ginecol Obstet Bras 1984; 7:125-31.

5. Rattner D. Sobre a hipótese de estabilização das taxas de cesárea do Estado de São Paulo, Brasil. Rev Saúde Pública 1996; 30:19-33.

6. Faúndes A, Cecatti JG. Which policy for caesarian sections in Brazil? An analysis of trends and consequences. Health Policy Plan 1993; 8:33-42.

7. Cunha AA, Portela MC, Amed AM, Camano L. Cesariana de repetição. Rev Ginecol Obstet 2000; 11:167-73.

8. Cunha AA. Avaliação epidemiológica dos fatores de risco anteparto para operação cesariana no Hospital Universitário Pedro Ernesto no período de junho de 1993 a novembro de 1994 [tese]. São Paulo: Univ. Federal de São Paulo; 1999.

9. Edwards C, Witter FR. Preeclampsia, labor duration and mode of delivery. Int J Gynaecol Obstet 1997; $57: 39-42$. 
10.North RA, Taylor RS, Schellenberg JC. Evaluation of a definition of pre-eclampsia. Br J Obstet Gynaecol 1999; 106:767-73.

11.Persad VL, Baskett TF, O'Connell CM, Scott HM. Combined vaginal-cesarean delivery of twin pregnancies. Obstet Gynecol 2001; 98:1032-7.

12. Corrêa MD. Cesariana: incidência ideal. Rev Méd Minas Gerais 1992; 2:30-3.

13.Hannah ME, Ohlsson A, Farine D, et al. Induction of labor compared with expectant management for prelabor rupture of the membranes at term. N Engl J Med 1996; 334:1005-10.

14. Shalev E, Peleg D, Eliyahu S, Nahum Z. Comparison of 12- and 72-hour expectant management of premature rupture of membranes in term pregnancies. Obstet Gynecol 1995; 85:766-8.

15. Ottervanger HP, Keirse MJ, Smit W, Holm JP. Controlled comparison of induction versus expectant care for prelabor rupture of the membranes at term. J Perinat Med 1996; 24:237-42.

16.Scholz HS, Haas J, Petru E. Do primiparas aged 40 years or older carry an increased obstetric risk? Prev Med 1999; 29:263-6.
17.Amini SB, Catalano PM, Dierker LJ, Mann LI. Births to teenagers: trends and obstetric outcomes. Obstet Gynecol 1996; 87:668-74.

18.Yuan W, Steffensen FH, Nielsen GL, Moller M, Olsen J, Sorensen HT. A population-based cohort study of birth and neonatal outcome in older primipara. Int J Gynaecol Obstet 2000; 68:113-8.

19.Seyb ST, Berka RJ, Socol ML, Dooley SL. Risk of cesarean delivery with elective induction of labor at term in nulliparous women. Obstet Gynecol 1999; 94:600-7.

20.Abu-Heija AT, Chalabi HE. Great grand multiparity: is it a risk? Int $J$ Gynaecol Obstet 1997; 59:213-6.

21.Montenegro R. Obstetrícia fundamental. $6^{\mathrm{a}} \mathrm{ed}$. Rio de Janeiro: Guanabara Koogan; 1992. p.597-624.

22.Neme B. Obstetrícia básica. $1^{\mathrm{a}}$ ed. São Paulo: Sarvier; 1994. p.753-6.

23.CDC-Centers for Disease Control \& Prevention. EpiInfo [computer program]. Version 6.04d. Atlanta: CDC; 2001.

\section{Publique em RBGO envie seu artigo !}
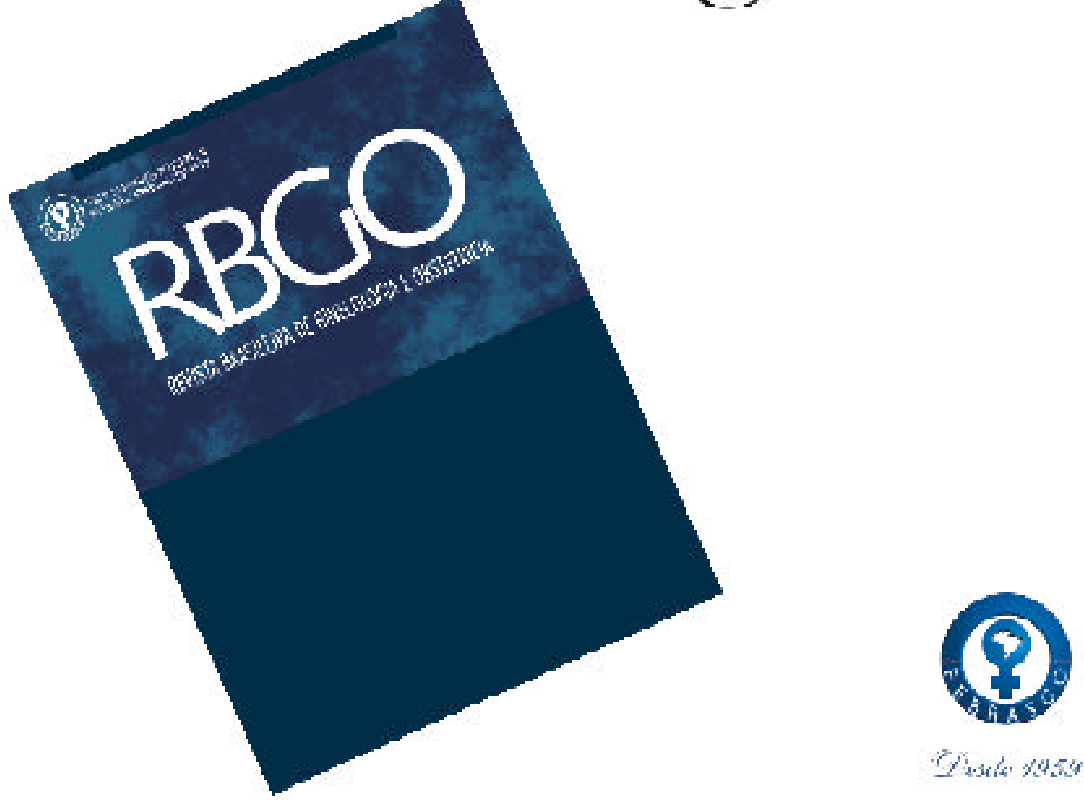